\title{
Diseño óptimo de cadenas de suministros reversas.
}

\section{Optimal reverse supply chain design.}

Presentación: 06-07/10/2020

\section{Doctorando:}

\section{Glenda N. Yossen}

Grupo de Investigación y Desarrollo en Informática Aplicada (GIDIA), Instituto de Desarrollo Tecnológico para la Industria Química (INTEC), Facultad Regional Santa Fe, Universidad Tecnológica Nacional - Argentina

gyossen@intec.unl.edu.ar

\section{Director/es:}

\section{Gabriela P. Henning}

\section{Co-director/es:}

\section{Resumen}

El creciente uso de agroquímicos sumado al daño que los envases contaminados causan al medio ambiente y a la salud de la población, hacen necesario establecer una cadena de suministros reversa para la recuperación y el correcto tratamiento de los envases. Se propone un modelo MILP multi-periodo para definir la configuración óptima de la cadena de suministros reversa de envases vacíos de agroquímicos, persiguiendo el objetivo de minimizar el costo total actualizado de toda la red.

Palabras clave: Logística reversa, Diseño y planificación de cadenas de suministros, Envases vacíos de agroquímicos, Modelo de programación mixta-entera lineal.

\begin{abstract}
The growing use of agrochemicals added to the harm that contaminated containers cause to the environment and to the health of the population, make it necessary to establish a reverse supply chain for the recovery and correct treatment of containers. A MILP multi-period model is proposed to define the optimal configuration of the reverse supply chain network of empty agrochemical containers, following the objective of minimizing the total actual cost of the network.
\end{abstract}

Keywords: Reverse logistics, Supply chain design and planning, Empty agrochemical containers, Mixed-integer linear programming model.

\section{Introducción}

Las cadenas de suministros actuales no se limitan a flujos de productos unidireccionales desde las fábricas hasta los consumidores. Por el contrario, deben lidiar con flujos de materiales opuestos con la finalidad de obtener la reutilización, reciclaje y/o disposición final de productos, piezas, así como envases de productos vacíos. La gestión de estos flujos reversos enfrenta a las empresas a nuevos desafíos que deben analizarse mediante la logística reversa. La misma puede definirse como el proceso que comprende la planificación, implementación y control de los flujos de retorno de materias primas, inventario en proceso, envases y productos terminados, desde un punto de fabricación, distribución o uso, hasta un punto de recupero o un punto adecuado de eliminación (Dekker et al, 2004).

Esta contribución aborda el diseño de una cadena de suministros reversa de envases de agroquímicos. Se pretende recolectar los envases plásticos desde los campos, una vez vaciados y triplemente lavados, con el objetivo de reciclar/utilizar el plástico, descontaminando el agua de lavado, minimizando los riesgos ambientales y sanitarios 
asociados. Las decisiones sobre la ubicación de las instalaciones desempeñan un rol fundamental en el diseño de las cadenas de suministros, como se señala en (Melo et al, 2009). El problema de configurar esta red en particular en un horizonte de planificación dado implica establecer: (i) el número y ubicación de los centros de acopio - puntos intermedios de almacenamiento de envases -, así como las plantas de tratamiento de plásticos a instalar, (ii) el periodo en que se instalará cada facilidad y en los que operará, (iii) las capacidades de las distintas instalaciones de almacenamiento y procesamiento, (iv) los flujos de envases para cada período de tiempo entre los campos y los puntos de acopio/tratamiento, así como los flujos entre los centros de acopio y las plantas de tratamiento de plásticos. Este problema se aborda por medio de un modelo MILP (Programación mixta entera lineal) multi-periodo que es capaz de abordar los desafíos presentados anteriormente, minimizando el costo total actualizado de la red.

\section{Desarrollo}

En el modelo MILP propuesto se asume que: (i) todos los parámetros del modelo son determinísticos, es decir, no se considera incertidumbre, (ii) los envases que se generan en un período dado deben ser recolectados y tratados en el mismo período, (iii) los centros de acopio y plantas de tratamiento que se instalarán son de capacidades discretas, (iv) los costos operativos variables se toman en cuenta sólo para las plantas de tratamiento de plásticos, (v) Los costos de transporte sólo consideran la distancia recorrida y omiten la carga de los vehículos.

\section{Nomenclatura}

Conjuntos

$f$
$j$
$p$
$m / n$
$t, t^{\prime}$
$j i / p i$

campos

centros de acopio

plantas de tratamiento

conjunto de tamaños posibles para centros de acopio/plantas de tratamiento periodos de tiempo

subconjunto de centros de acopio/plantas de tratamiento que se encuentran instalados al inicio del horizonte de planificación

Parámetros

$g_{f t}$

$d_{f j} / d_{f p} / d_{j p}$

$k_{m}^{\min } / k_{n}^{\min }$

$k_{m}^{\max } / k_{n}^{\max }$

nt j /nt p

$c^{f j} / \mathrm{cfp} / \mathrm{cjp}$

ir

$f c_{m} / f c_{n}$

$\mathrm{vc}$

$c c_{m}^{j} / c c_{n}^{p}$

$e c_{n}$

cap

$n f_{f t}$ cantidad de envases vacíos generados por el campo f en el periodo $t$

distancia entre los distintos tipos de nodos

capacidad mínima de un centro de acopio/planta de tratamiento de tamaño $\mathrm{m} / \mathrm{n}$ capacidad máxima de un centro de acopio/planta de tratamiento de tamaño $\mathrm{m} / \mathrm{n}$ number of periods required by a new collection center/treatment plant to start operating costo de transporte por unidad de distancia entre los diferentes nodos de la red tasa de interes anual costo fijo de operación de un centro de acopio/planta de tratamiento de tamaño $\mathrm{m} / \mathrm{n}$ costo variable de operación de plantas de tratamiento costo de construcción de un centro de acopio/planta de tratamiento de tamaño $\mathrm{m} / \mathrm{n}$ costo del equipamiento requerido por una planta de tratamiento de tamaño $\mathrm{n}$ capacidad de carga de los camiones utilizados para transportar envases desde centros de acopio a plantas de tratamiento

cantidad de viajes requeridos para transportar la generación total de envases del campo f en el periodo $\mathrm{t}$

Variables de decisión

Variables binarias

$X_{j m} / X_{p n} \quad$ vale uno el centro de acopio j/la planta de tratamiento $\mathrm{p}$ se instala de tamaño $\mathrm{m} / \mathrm{n}$

$Y_{j t} / Y_{p t} \quad$ vale uno si el centro de acopio $\mathrm{j} /$ la planta de tratamiento $\mathrm{p}$ opera en el periodo $\mathrm{t}$

$I_{j t} / I_{p t} \quad$ vale uno si el centro de acopio j/la planta de tratamiento $\mathrm{p}$ se instala en el periodo $\mathrm{t}$

Variables positivas

$S_{f j t} / S_{f p t} / S_{j p t}$ cantidad de envases enviada entre los diferentes tipos de nodos en el periodo $\mathrm{t}$ 


\section{Formulación MILP}

Utilizando las definiciones anteriores, el modelo se formula de la siguiente manera.

$$
\begin{aligned}
& g_{f t}=\sum_{j} S_{f j t}+\sum_{p} S_{f p t} \quad \forall f, \forall t \\
& \sum_{f} S_{f j t}=\sum_{p} S_{j p t} \quad \forall j, \forall t \\
& \sum_{f} g_{f t}=\sum_{f, p} S_{f p t}+\sum_{j, p} S_{j p t} \quad \forall t \\
& \sum_{t^{\prime}+n t^{j} \leq t} I_{j t^{\prime}} \geq Y_{j t} \quad \forall j, \forall t \\
& \sum_{t^{\prime}+n t^{p} \leq t} I_{p t^{\prime}} \geq Y_{p t} \quad \forall p, \forall t \\
& \sum_{m} X_{j m}=\sum_{t} I_{j t} \quad \forall j \\
& \sum_{n} X_{k n}=\sum_{t} I_{k t} \quad \forall k \\
& \sum_{m} X_{j m} \leq 1 \quad \forall j, \forall t \\
& \sum_{n} X_{p n} \leq 1 \quad \forall p, \forall t \\
& \sum_{f} S_{f j t} \leq\left(k_{m=1}^{\max } \cdot Y_{j t}\right)+\sum_{m>1}\left(k_{m}^{\max }-k_{1}^{\max }\right) \cdot X_{j m} \quad \forall j, \forall t \\
& \sum_{f} S_{f p t}+\sum_{j} S_{j p t} \leq\left(k_{n=1}^{\max } \cdot Y_{p t}\right)+\sum_{n>1}\left(k_{n}^{\max }-k_{n=1}^{\max }\right) \cdot X_{p n} \quad \forall p, \forall t \\
& \sum_{f} S_{f j t} \geq\left(k_{m=1}^{\min } \cdot Y_{j t}\right)+\sum_{m>1}\left(k_{m}^{\min }-k_{1}^{\min }\right) \cdot X_{j m} \quad \forall j, \forall t \\
& \sum_{f} \mathrm{~S}_{\mathrm{fpt}}+\sum_{j} \mathrm{~S}_{\mathrm{jpt}} \geq\left(\mathrm{k}_{\mathrm{n}=1}^{\min } \cdot \mathrm{Y}_{\mathrm{pt}}\right)+\sum_{n>1}\left(\mathrm{k}_{n}^{\min }-\mathrm{k}_{\mathrm{n}=1}^{\min }\right) \cdot \mathrm{X}_{\mathrm{pn}} \quad \forall p, \forall t \\
& I c c_{t}=\sum_{j m} c c_{m}^{j} \cdot\left(I_{j t}+X_{j m}-1\right) \quad \forall t \\
& I c p_{t}=\sum_{p n}\left(c c_{n}^{p}+e c_{n}\right) \cdot\left(I_{p t}+X_{p n}-1\right) \quad \forall t
\end{aligned}
$$




$$
\begin{gathered}
V c p_{t}=\sum_{p} v c \cdot\left(\sum_{f} S_{f p t}+\sum_{j} S_{j p t}\right) \quad \forall t \\
O c j_{t}=\sum_{j}\left[\left(Y_{j t} \cdot f c_{m=1}\right)+\sum_{m} X_{j m} \cdot\left(f c_{m}-f c_{1}\right)\right] \quad \forall t \\
O c p_{t}=\sum_{p}\left[\left(Y_{p t} \cdot f c_{n=1}\right)+\sum_{n} X_{p n} \cdot\left(f c_{n}-f c_{n=1}\right)\right] \quad \forall t \\
T c^{f j}{ }_{t}=\sum_{f, j}\left(c^{f j} 2 d_{f j} \frac{S_{f j t}}{g_{f t}} \cdot n f_{f t}\right) \quad \forall t \\
T c^{f p}{ }_{t}=\sum_{f, p}\left(c^{f p} 2 d_{f p} \frac{S_{f p t}}{g_{f t}} \cdot n f_{f t}\right) \quad \forall t \\
T c_{t}^{j p}=\sum_{j, p}\left(c^{j p} 2 d_{j p} 1.2 \frac{S_{f p t}}{c a p}\right) \quad \forall t \\
\sum_{t} \frac{\forall c c_{t}+I c p_{t}+V c p_{t}+O c j_{t}+O c p_{t}+T c^{f j}{ }_{t}+T c^{f p}{ }_{t}+T c^{j p}{ }_{t}}{(1+i r)^{t}}
\end{gathered}
$$

La Ec. (1) establece que todos los envases generados por un campo en un período determinado, deben ser enviados a un centro de acopio y/o planta de tratamiento en el mismo período. La Ec. (2) establece que todos los envases que son recibidos por un centro de acopio en un período determinado deben ser enviados a una planta de tratamiento en el mismo período. De manera similar, la Ec. (3) especifica que el número total de contenedores vacíos recibidos por todas las plantas de tratamiento desde los campos y/o desde los centros de acopio durante un período determinado debe ser igual al número total de contenedores vacíos generados por todos los campos en dicho período. La Ec. (4) asegura que un centro de acopio puede operar en un período determinado sólo si se ha instalado en uno anterior. La Ec. (5) obliga a las plantas de tratamiento a obedecer la misma restricción.

La Ec. (6) establece que, si se instala un centro de acopio en un período determinado, debe adoptar uno de los $\mathrm{m}$ tamaños disponibles. De manera similar, la Ec. (7) prescribe que, si se instala una planta de tratamiento de plásticos en el período t, debe ser de uno de los n tamaños disponibles. Las Ecs. (8) y (9) obligan a que cada centro de acopio/planta de tratamiento que se instale debe adoptar sólo uno de los tamaños disponibles. De acuerdo a la Ec. (10), el número de contenedores enviados a un centro de acopio determinado en cada período, no debe superar la capacidad máxima de almacenamiento correspondiente al tamaño instalado. La capacidad máxima de cada planta de tratamiento a instalar se captura mediante la Ec. (11). La capacidad mínima de cada centro de acopio o planta de tratamiento de plásticos a instalar viene dada por las expresiones (12) y (13), respectivamente.

Las Ecs. (14) - (17) capturan los diferentes términos de costo que participan en la función objetivo. La Ec. (14) establece el costo de inversión de los nuevos centros de acopio en cada período, Icc (t). Cabe señalar que, si se instala un centro de acopio antes del inicio del horizonte de planificación $(\mathrm{j} \in \mathrm{ji}$ ), entonces su costo es cero. De manera similar, la Ec. (15) captura el costo de inversión de nuevas plantas de tratamiento en cada período, considerando tanto el costo de construcción como el costo del equipo asociado con el tamaño de la planta a instalar. Cabe señalar que estos costos se calculan aplicando economías de escala.

Los costos variables por período sólo se consideran para las plantas de tratamiento de plásticos, como se muestra en la Ec. (16), considerando tanto el costo unitario de procesamiento como el número total de envases enviados a cada planta en cada período.

El costo fijo de operación asociado a los centros de acopio en cada período viene dado por la Ec. (17) y es dependiente del tamaño adoptado m. De manera similar, la Ec. (18) establece el costo fijo de operación de las plantas de tratamiento en cada período, dependiente del tamaño $\mathrm{n}$ que se adopte para cada una.

Los costos de transporte de los campos a los centros de acopio para cada uno de los períodos de planificación son capturados por Ec. (19). Mediante esta expresión, se consideran los viajes de ida y vuelta multiplicando el costo de transporte por unidad de distancia desde el campo hasta el centro de acopio j, por el doble de la distancia entre estos 
nodos. Esto, a su vez, se multiplica por la proporción del número de viajes necesarios para transportar la generación total de envases de cada campo a los centros de acopio. Cabe señalar que la cantidad de viajes requeridos se calcula considerando 1,5 veces el número real de viajes que se requerirían si se hubiera empleado la capacidad completa del vehículo. Esta suposición se basa en el hecho de que la mayoría de los viajes se realizan con menos de la carga máxima permitida. La proporción se calcula dividiendo el número de contenedores enviados desde cada campo a cada centro de acopio en un período dado, por la generación de contenedores del campo en dicho período. El costo de transporte de los campos a las plantas de tratamiento, para cada período de planificación, es capturado por Ec. (20) de la misma forma en la que se ha estimado el costo de transporte entre campos y centros de acopio. Finalmente, el costo de transporte de los centros de acopio a las plantas de tratamiento, para cada período de planificación, se estima mediante la Ec. (21). En este caso, se considera 1,2 veces el número total de viajes requeridos, ya que se puede llevar a cabo una mayor consolidación de envases. Considerando estos componentes de costos, la función objetivo viene dada por la Ec. (22). La tasa de interés permite calcular el costo total descontado de la red.

\section{Resultados}

El modelo ha sido implementado en GAMS 23.6, ejecutándose en una computadora Intel Core i3, $2.5 \mathrm{GHz}, 4 \mathrm{~GB}$ de RAM, utilizando el solver CPLEX.

Para probar la respuesta del modelo a cambios en las condiciones y analizar su escalabilidad, se han resuelto escenarios adicionales utilizando diferentes conjuntos de datos. Cabe mencionar que, debido a la dimensión de los modelos, en los cinco primeros escenarios el tiempo computacional se restringió a $3600 \mathrm{~s}$, mientras que en los escenarios 5 y 6 se extendió a 7200 s de CPU. La Tabla 1 resume algunos de los escenarios que se han abordado.

\begin{tabular}{ccccc}
\hline & Campos & $\begin{array}{c}\text { Centros de } \\
\text { acopio }\end{array}$ & $\begin{array}{c}\text { Plantas de } \\
\text { tratamiento }\end{array}$ & $\begin{array}{c}\text { Cantidad de } \\
\text { periodos }\end{array}$ \\
\hline Caso Base & 300 & 20 & 8 & 6 \\
Escen. 1 & 600 & 20 & 8 & 6 \\
Escen. 2 & 300 & 20 & 8 & 12 \\
Escen. 3 & 300 & 20 & 16 & 6 \\
Escen. 4 & 300 & 40 & 8 & 6 \\
Escen. 5 & 300 & 40 & 16 & 6 \\
Escen. 6 & 600 & 40 & 16 & 6
\end{tabular}

Tabla 1: Resumen de los escenarios analizados.

En la Tabla 2 se detallan, para cada uno de los escenarios analizados, la cantidad total de variables resultante de la implementación del modelo matemático, la cantidad de variables binarias, el total de restricciones. El valor de la función objetivo obtenido, como así también el tiempo de CPU utilizado (en segundos) y el GAP relativo obtenido.

\begin{tabular}{ccccccc}
\hline & $\begin{array}{c}\text { Total } \\
\text { variables }\end{array}$ & $\begin{array}{c}\text { Variables } \\
\text { binarias }\end{array}$ & $\begin{array}{c}\text { Total } \\
\text { restricciones }\end{array}$ & $\begin{array}{c}\text { Valor de la } \\
\text { FO }\end{array}$ & CPUs & $\begin{array}{c}\text { Gap } \\
\text { relativo }\end{array}$ \\
\hline Caso Base & 52,189 & 420 & 3,511 & $1,463,195.55$ & 501 & 0,00000 \\
Escen. 1 & 102,253 & 420 & 5,004 & $1,940,265.99$ & 3000 & 0,07716 \\
Escen. 2 & 103,621 & 756 & 6,378 & $1,663,211.04$ & 3600 & 0,02074 \\
Escen. 3 & 103,999 & 540 & 6,756 & $1,393,630.05$ & 3600 & 0,02006 \\
Escen. 4 & 89,113 & 720 & 4,184 & $1,473,526.940$ & 3600 & 0,02994 \\
Escen. 5 & 105,273 & 840 & 5,192 & $1,021,613.940$ & 7056 & 0,00000 \\
Escen. 6 & 207,073 & 840 & 6,992 & $2,491,705.037$ & 7173 & 0,00000
\end{tabular}

Tabla 2: Resultados computacionales de los diferentes escenarios analizados.

En la Tabla 3 se presenta un resumen de las facilidades a instalar para cada uno de los escenarios, detallando además sus correspondientes tamaños. 


\begin{tabular}{ccccc}
\hline & $\begin{array}{c}\text { Centros } \\
\text { de acopio }\end{array}$ & $\begin{array}{c}\text { Plantas de } \\
\text { tratamiento }\end{array}$ & $\begin{array}{c}\text { Tamaño de los } \\
\text { centros de acopio }\end{array}$ & $\begin{array}{c}\text { Tamaño de las plantas } \\
\text { de tratamiento }\end{array}$ \\
\hline Caso Base & 1 & 2 & pequeño & ambas pequeñas \\
Escen. 1 & 1 & 2 & pequeño & 1 pequeña, 1 mediana \\
Escen. 2 & 2 & 2 & 1 pequeño, 1 mediano & ambas pequeñas \\
Escen. 3 & 1 & 2 & pequeño & ambas pequeñas \\
Escen. 4 & 2 & 2 & ambos pequeños & ambas pequeñas \\
Escen. 5 & 1 & 2 & pequeño & ambas pequeñas \\
Escen. 6 & 1 & 3 & pequeño & 2 pequeñas, 1 mediana
\end{tabular}

Tabla 3: Soluciones de los escenarios al final del horizonte de planificación.

Para el caso base la solución óptima implica la instalación de un centro de acopio y dos plantas de tratamiento en el primer año, todos de la menor capacidad entre las disponibles. El escenario 1 duplica el número de campos con respecto al caso base, pero mantiene constante el número de centros de acopio y plantas de tratamiento posibles. La solución obtenida comprende el mismo número de instalaciones que el caso base. Sin embargo, se instala una de las plantas de tratamiento de mayor tamaño, con una capacidad máxima de procesamiento de 100.000 envases anuales, para poder atender la generación de un mayor número de campos. Otra diferencia importante entre estas soluciones es el hecho de que el centro de acopio se instala durante el segundo año y se coloca en un lugar diferente. El escenario 2 tiene en cuenta la misma superestructura que el caso base, pero duplica la longitud del horizonte de planificación. La solución en este caso comprende cuatro instalaciones: 2 centros de acopio y 2 plantas de tratamiento, las cuales se instalan durante el período inicial. Esto demuestra que el efecto de considerar un horizonte de planificación más extenso es el incremento en la inversión en la instalación de centros de acopio, con el objetivo de reducir los costos de transporte. El escenario 3 duplica la cantidad de plantas de tratamiento posibles con respecto al caso base, manteniendo los restantes conjuntos sin alterar. La solución obtenida es similar a la obtenida en el caso base, pero con un menor costo total de la red, debido a que se provee al modelo de una mayor superestructura, lo que otorga mayores posibilidades de configuración de la red. El escenario 4 duplica la cantidad de centros de acopio posibles, manteniendo los demás conjuntos constantes respecto al caso base. La solución obtenida es nuevamente similar a la del caso base, con un GAP del 3 en 3600 s de CPU. Los escenarios 3 y 4 permiten concluir que cuanto mayor sea el número de nodos potenciales incluidos en el modelo, mayores serán las posibilidades de obtener soluciones de buena calidad. Además, se verifica que el incremento en el número de variables binarias también demanda un esfuerzo computacional adicional. Los escenarios 5 y 6, por su parte, no solo duplican el número de posibles centros de acopio con respecto al caso base, sino también el número de posibles plantas de tratamiento. Además, el escenario 6 duplica el número de campos considerados. La solución correspondiente al escenario 5 comprende 3 facilidades: dos plantas de tratamiento y un centro de acopio, todas del menor tamaño dentro de los disponibles. Para hacer frente a una mayor generación de envases, el escenario 6 da como resultado una cadena de suministros compuesta por 4 instalaciones: un centro de acopio y tres plantas de tratamiento, una de las cuales tiene capacidad media, pudiendo procesar hasta 100.000 envases por año. Mientras que en el escenario 5 todas las facilidades se instalan durante el período inicial, la solución del escenario 6 incluye tres instalaciones durante el período inicial y una planta de tratamiento que se instala en el período 3, con el fin de responder a un aumento simulado en la generación de contenedores asociada al período 4.

La aplicación del modelo muestra que se obtienen soluciones óptimas o sub-óptimas de buena calidad en tiempos de CPU bajos, considerando un problema de alcance estratégico. Los costos de mayor incidencia en todos los casos son aquéllos asociados a la instalación de facilidades y al transporte entre las mismas.

Como trabajo futuro, se realizarán más pruebas del modelo para analizar en profundidad los problemas de escalabilidad. Por ejemplo, se abordará un horizonte temporal con períodos de menor duración y una superestructura con más nodos. Además, se evaluará la incorporación de objetivos ambientales y sociales, en una situación de compromiso con los económicos.

\section{Referencias}

Dekker R., Fleischmann M., Inderfurth K., Van Wassenhove L. (2004). Reverse Logistics Quantitative models for closedloop supply chains. Berlin: Springer Verlag.

GAMS, A user's Guide. https://www.gams.com/latest/docs/UG_MAIN.html.

Melo M., Nickel S., Saldanha-da-Gama F. (2009). Facility location and supply chain management- A review. Eur. J. Oper. Res. 196, 401-412. doi: 10.1016/j.ejor.2008.05.07. 